\title{
Thrombocytapheresis in Patient with Essential Thrombocythemia: A Case Report
}

\author{
Afra M. Elhassan ${ }^{a} \quad$ Arwa Alsaud $^{\mathrm{a}}$ Mohamed A. Yassin ${ }^{\mathrm{b}}$ \\ Mahmood Aldapt ${ }^{b} \quad$ Lubna Riaz $^{b}$ Firdous Ghori ${ }^{b} \quad$ Aiman Bin Ahmad ${ }^{b}$ \\ Mohammad Abdullab \\ aDepartment of Internal Medicine, Hamad Medical Corporation, Doha, Qatar; ${ }^{\text {b National }}$ \\ Centre for Cancer Care and Research, Hamad Medical Corporation, Doha, Qatar
}

\author{
Keywords \\ Platelet · Plateletpheresis · Thrombocytapheresis · Thrombocytosis · Essential \\ thrombocythemia
}

\section{Abstract}

Essential thrombocythemia (ET) is one of the myeloproliferative neoplasms, characterized by persistent thrombocytosis, platelets $>450,000 / \mu \mathrm{L}$, and evident clonal abnormalities like JAK2 V617F, MPL, CALR mutation and not fulfilling WHO criteria for MDS, CML, PV, and IDA. Here we report a 24-year-old female who presented with headache and was found to have thrombocytosis with a platelet count of 2,141 $\times 10^{3} / \mu \mathrm{L}$, diagnosed as ET as per WHO criteria 2008; she required ICU admission and thrombocytapheresis with a favorable outcome.

(c) 2020 The Author(s).

Published by S. Karger AG, Basel

\section{Introduction}

Essential thrombocythemia (ET) is one of the myeloproliferative neoplasms (MPNs), characterized by a persistent platelet count of $\geq 450 \times 10^{9} / \mathrm{L}$, bone marrow showing proliferation mainly of the megakaryocyte lineage with increased numbers of enlarged, mature megakaryocytes with hyperlobulated nuclei, presence of JAK2, CALR, or MPL mutation, not

Dr. Afra Elhassan and Dr. Mohamed Yassin equally contributed to this study. 
meeting WHO criteria for BCR-ABL1+ CML, PV, PMF, myelodysplastic syndromes, or other myeloid neoplasms [1].

There has been a lack of estimates for the incidence/prevalence and treatment patterns of MPNs in many regions of the world, including countries from South Asia, Asia Pacific, Middle East, and Turkey. Yassin et al. [2] investigated 884 MPN patients from these countries. The prevalence and incidence of MPNs were found to be 57-81 and 12-15 per 100,000 hospital patients per year over the last 4 years, respectively.

The goals of ET management are to alleviate symptoms and minimize complications of the disease (e.g., thrombotic events, bleeding) [3]. Patients with extreme thrombocytosis and hemorrhage should be treated to lower the platelet count with cytoreduction therapy, either chemically or through thrombocytapheresis [4].

Most patients with ET enjoy a normal life expectancy, although survival is affected by disease features used for risk stratification [5]. In a multinational study conducted in adult patients with MPNs in Asia, including Middle East, Turkey, and Algeria, despite being on treatment, patients with MPN had a substantial symptom burden, which did not change over the course of the study [6]. However, this is applied to sporadic cases and familial cases from different parts of the world were included in different studies [7].

In this case report, we are highlighting the treatment options and their rationale of selection.

\section{Case Report}

We report a 24-year-old Egyptian woman, who presented to our emergency department complaining of severe headache. She had previously been diagnosed with ET 6 months ago following extensive investigations for thrombocytosis as per the WHO criteria 2008 and was kept under close observation. She had remained asymptomatic since that time until this visit. Upon her presentation, she denied any neurologic deficit or associated symptoms. Her vitals and clinical examination were unremarkable apart from splenomegaly.

Lab investigation showed a platelet count of $2,142 \times 10^{3} / \mu \mathrm{L}(150-400)$, WBC count of 4.3 $\times 10^{3} / \mu \mathrm{L}(4-10)$, RBC count of $2.8 \times 10^{6} / \mu \mathrm{L}(3.8-4.8), \mathrm{Hb}$ of $9.1 \mathrm{~g} / \mathrm{dL}(12-15)$, hematocrit $28.4 \%$ (36-46), and MCV 99.5 fL (83-101). Emergency CT scan of the head was done and showed no obvious intracerebral or subarachnoid hemorrhage, no obvious infarction, ventricles in normal position and size and CSF spaces looked normal.

A previous test showed a platelets count of 3,175 × 103/ $\mathrm{LL}(150-400)$, peripheral smear had marked thrombocytosis, many large forms and few atypical (reactive) lymphocytes. Her bone marrow showed hypercellular marrow with remarkable proliferation of megakaryocytes, with overall morphological findings consistent with ET. A diagnosis of ET was confirmed after exclusion of iron deficiency anemia and because all genetic mutations including JAK2 V617F, MPL, CALR were negative.

The decision was made to admit her to medical ICU for plateletpheresis. A femoral line was inserted, and she underwent 2 sessions of thrombocytapheresis. Fortunately, her platelet count dropped significantly, and her symptoms resolved (Fig. 1).

She was started on cytoreduction therapy with hydroxyurea. Later she developed blackish discoloration in her hand from hydroxyurea, which she did not tolerate, so it was stopped. We started her on pegylated interferon-alfa with a dose of $135 \mu \mathrm{g}$ subcutaneously per week. She continued this regimen and her platelet count continued to drop (Fig. 2).

Currently, she is asymptomatic on the same dose of pegylated interferon, tolerating it very well, and she successfully conceived twice and had two healthy baby boys. 


\section{platelet count drop after thrombocytapheresis}

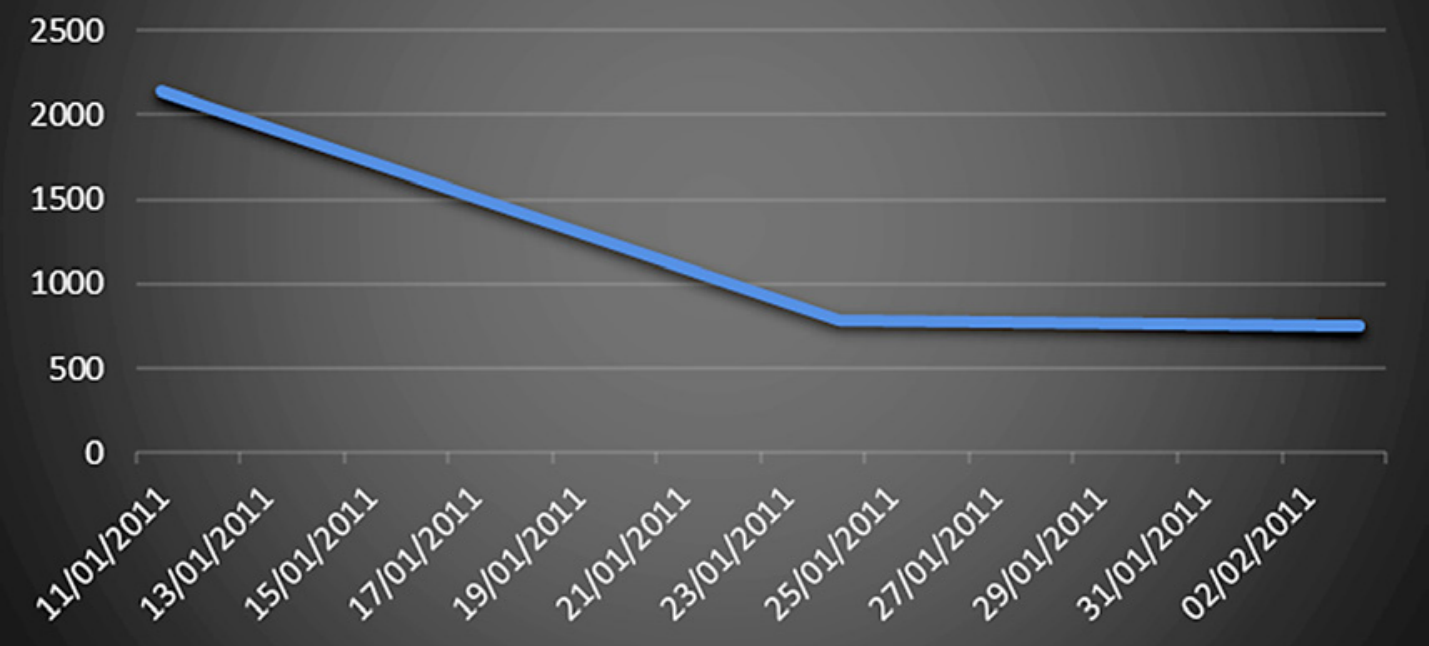

Fig. 1. Platelet count drop after thrombocytapheresis.

\section{platelet count after introduction of Pegylated Interferon alfa}

1200

1000

800

600

400

200

0

$18 / 08 / 2011 \quad 18 / 09 / 2011 \quad 18 / 10 / 2011 \quad 18 / 11 / 2011 \quad 18 / 12 / 2011$

Fig. 2. Platelet count after introduction of pegylated interferon-alfa. 
Elhassan et al.: ET and Thrombocytapheresis

\section{Discussion}

Our patient's presentation met the WHO criteria for ET, although thrombocytosis itself is not an indication for cytoreduction therapy; however, patients with platelets $>1$ million $/ \mu \mathrm{L}$ are at a high risk of developing acquired von Willebrand syndrome. Therefore, the decision of thrombocytapheresis was made based on the high platelet count and the risk of intracerebral hemorrhage.

The decision whether to start treatment or not and even the type of therapy depends on the calculated risk score for individual patients using the IPSET-thrombosis model, based on patient age, history of thrombosis, presence of JAK2 V617F mutation, and cardiovascular risk factors. Patients with low- or very-low-risk IPSET-thrombosis score are recommended to be treated with low-dose aspirin or even observation alone. On the other hand, for those with intermediate to high-risk scores, the recommendation is cytoreduction therapy with or without systemic anticoagulation or low-dose aspirin [3].

Cytoreduction therapy could be pharmacological or nonpharmacological. Hydroxyurea is considered the first-line pharmacological cytoreduction therapy, but due to its side effects, many patients do not tolerate it. Pegylated interferon-alfa, due to its nonleukemogenic effect, and a more favorable toxicity profile are the preferred second-line cytoreduction therapy, specially for younger patients and those who want to become pregnant [8-10]. Thrombocytapheresis has been utilized to prevent recurrent or to treat acute thromboembolism or hemorrhage in selected patients with MPN and uncontrolled thrombocytosis. It has also been used to treat extreme rebound thrombocytosis after splenectomy and, during pregnancy, to prevent recurrent fetal loss in high-risk patients with PV or ET. Thrombocytapheresis is indicated in patient with a platelet count $>1$ million $/ \mu \mathrm{L}$ as they are prone to develop acquired von Willebrand syndrome. It aids in restoring normal platelet count and corrects the short plasma half-life of large von Willebrand factor multimers with ET. Elective thrombocytapheresis should also be considered for cytoreduction of patients at increased risk of major hemorrhage when hydroxyurea is contraindicated, such as in pregnancy or in situations when the onset of action of hydroxyurea cytoreduction is too slow. Each procedure lowers the platelet count by $30-60 \%[4]$.

\section{Conclusion}

Despite the effectiveness of cytapheresis, still chemical cytoreduction, specially hydroxyurea, is being used worldwide. This is largely due to the high cost of cytapheresis therapy and the need of a special setting that may not be available everywhere.

\section{Acknowledgement}

We thank the Department of Internal Medicine in Hamad Medical Corporation, especially Dr. Ahmed Al-Mohammed and Dr. Dabia Al Mohanadi, for their support and motivation. Also, we thank Qatar National Library for the resources and opportunities.

\section{Statement of Ethics}

This work was conducted ethically in accordance with the World Medical Association Declaration of Helsinki. Informed consent was taken from the patient to publish her case. 
Elhassan et al.: ET and Thrombocytapheresis

\section{Disclosure Statement}

The authors have no conflicts of interest to declare.

\section{Funding Sources}

This work was supported by Qatar National Library.

\section{Author Contributions}

Afra M. Elhassan: Literature review and manuscript writing. Arwa Alsaud: Assisting in manuscript writing. Mohamed A. Yassin: Literature review and manuscript editing (Mentor). Mahmood Aldapt, Lubna Riaz, Firdous Ghori, Aiman Bin Ahmed, Mohammad Abdulla: Clinical management.

\section{References}

1 Arber DA, Orazi A, Hasserjian R, Thiele J, Borowitz MJ, Le Beau MM, et al. The 2016 revision to the World Health Organization classification of myeloid neoplasms and acute leukemia. Blood. 2016;127(20):2391-405.

2 Yassin M, Taher A, Mathews V, Hou H, Shamsi T, Tuğlular T, et al. A Multinational, Multicenter Observational Registry for Myeloproliferative Neoplasms in Asia, including Middle East, Turkey, and Algeria. Cancer Med. 2020 Apr;30. 10.1002/cam4.3004.

3 Barosi G, Mesa R, Finazzi G, Harrison C, Kiladjian JJ, Lengfelder E, et al. Revised response criteria for polycythemia vera and essential thrombocythemia: an ELN and IWG-MRT consensus project. Blood. 2013; 121(23):4778-81.

4 Schwartz J, Padmanabhan A, Aqui N, Balogun RA, Connelly-Smith L, Delaney M, et al. Guidelines on the Use of Therapeutic Apheresis in Clinical Practice-Evidence-Based Approach from the Writing Committee of the American Society for Apheresis: The Seventh Special Issue. J Clin Apher. 2016;31(3):149-62.

5 Passamonti F, Rumi E, Pungolino E, Malabarba L, Bertazzoni P, Valentini M, et al. Life expectancy and prognostic factors for survival in patients with polycythemia vera and essential thrombocythemia. Am J Med. 2004; 117(10):755-61.

6 Ali T, MA Yassin, Z Xiao, H-A Hou, T Tuglular, V Mathews, et al. Impact of myeloproliferative neoplasms (MPNs) on health-related quality of life (HRQOL) and medical resource utilization: results from the MERGE registry. Blood, 2018;32(Suppl 1):4311.

7 Al-Dewik N, Ben-Omran T, Zayed H, Trujillano D, Kishore S, Rolfs A, et al. Clinical Exome Sequencing unravels new disease-causing mutations in the myeloproliferative neoplasms: A pilot study in patients from the state of Qatar. Gene. 2019;689:34-42.

8 Yassin M, Al-Dewik N, El-Ayoubi H. Pegylated interferon-alfa-2a induces complete hematologic and molecular responses with low toxicity in essential thrombocythemia. Blood. 2012;120(21):1734. https://doi. org/10.1182/blood.V120.21.1743.1743.

9 Yassin M, Al-Dewik N, ElAyoubi H, Cassinat B. Efficacy and Safety of Pegelated Interferon alpha2a once Monthly Compared To Once Weekly Dose In Patients With Essential thrombocythemia. Blood. 2013;21:1224054. https://doi.org/10.1182/blood.V122.21.4054.4054.

10 Verger E, Cassinat B, Chauveau A, Dosquet C, Giraudier S, Schlageter M-H, et al. Clinical and molecular response to interferon- $\alpha$ therapy in essential thrombocythemia patients with CALR mutations. Blood. 2015; 126(24):2585-91. 10.1182/blood-2015-07-659060. 26486786. 